\title{
Bacterial Protein-Tyrosine Kinases
}

Shi, Lei; Kobir, Ahasanul; Jers, Carsten; Mijakovic, Ivan

\section{Published in:}

Current Proteomics

Publication date:

2010

Link back to DTU Orbit

Citation (APA):

Shi, L., Kobir, A., Jers, C., \& Mijakovic, I. (2010). Bacterial Protein-Tyrosine Kinases. Current Proteomics, 7(3, Sp. Iss. SI), 188-194.

\section{General rights}

Copyright and moral rights for the publications made accessible in the public portal are retained by the authors and/or other copyright owners and it is a condition of accessing publications that users recognise and abide by the legal requirements associated with these rights.

- Users may download and print one copy of any publication from the public portal for the purpose of private study or research.

- You may not further distribute the material or use it for any profit-making activity or commercial gain

- You may freely distribute the URL identifying the publication in the public portal

If you believe that this document breaches copyright please contact us providing details, and we will remove access to the work immediately and investigate your claim. 


\title{
Bacterial Protein-Tyrosine Kinases
}

\author{
Lei Shi ${ }^{1}$, Ahasanul Kobir ${ }^{1}$, Carsten Jers ${ }^{2}$ and Ivan Mijakovic ${ }^{*}, 1$ \\ ${ }^{I}$ Micalis UMR 1319, AgroParisTech-INRA, Domaine de Vilvert, 78352 Jouy-en-Josas, France France; ${ }^{2}$ Center for Sys- \\ tems Microbiology, Technical University of Denmark, DK-2800 Lyngby, Denmark
}

\begin{abstract}
Bacteria and Eukarya share essentially the same family of protein-serine/threonine kinases, also known as the Hanks-type kinases. However, when it comes to protein-tyrosine phosphorylation, bacteria seem to have gone their own way. Bacterial protein-tyrosine kinases (BY-kinases) are bacterial enzymes that are unique in exploiting the ATP/GTPbinding Walker motif to catalyze phosphorylation of protein tyrosine residues. Characterized for the first time only a decade ago, BY-kinases have now come to the fore. Important regulatory roles have been linked with these enzymes, via their involvement in exopolysaccharide production, virulence, DNA metabolism, stress response and other key functions of the bacterial cell. BY-kinases act through autophosphorylation (mainly in exopolysaccharide production) and phosphorylation of other proteins, which have in most cases been shown to be activated by tyrosine phosphorylation. Protein-tyrosine phosphorylation in bacteria is particular with respect to very low occupancy of phosphorylation sites in vivo; this has represented a major challenge for detection techniques. Only the recent breakthroughs in gel-free high resolution mass spectrometry allowed the systematic detection of phosphorylated tyrosines by phosphoprotomics studies in bacteria. Other pioneering studies conducted in recent years, such as the first structures of BY-kinases and biochemical and phyiological studies of new BY-kinase substrates significantly furthered our understanding of these enzymes and highlighted their importance in bacterial physiology. Having no orthologues in Eukarya, BY-kinases are receiving a growing attention from the biomedical field, since they represent a particularly promising target for anti-bacterial drug design.
\end{abstract}

Keywords: Protein-tyrosine kinase / BY-kinase / bacteria / protein phosphorylation / phosphoproteome / signal transduction / cellular regulation

\section{BY-KINASES IN BACTERIAL PHYSIOLOGY}

The literature in the research field of post-translational modifications of proteins abounds with statements that elaborate modifications found in Eukarya do not exist in Bacteria. On more than one occasion, this simply reflected the fact that nobody had made a serious effort looking for them. Recent examples of "missing" modifications finally found in bacteria are those of protein glycosylation [1], acetylation [2] and methylation [2]. In much the same spirit, protein-tyrosine phosphorylation was considered nonexistant in bacteria, until the pioneering studies appeared in the mid nineties $[3,4]$, identifying phosphorylated tyrosine residues in bacterial proteins. These first studies reported members of a new family of proteins that autophosphorylated on tyrosine, which were later named BY-kinases (for bacterial tyrosine kinases) [5], but are also known as polysaccharide copolymerases [6] due to multiple roles they play in the cellular physiology. BY-kinase-encoding genes have since been identified in a majority of sequenced bacterial genomes, and are thus considered widespread, if not ubiquitous, in the bacterial kingdom [7]. BY-kinases account for most tyrosine phosphorylation events identified in bacteria so far, the exception being some unusual two-component systems that phosphorylate on tyrosine. A typical BY-kinase

\footnotetext{
*Address correspondence to this author at the Micalis UMR 1319, AgroParisTech-INRA, F-78850 Thiverval-Grignon, FRANCE,

Tel: +33(0)1308145 40, Fax: +33(0)1 308154 57;

E-mail: Ivan.Mijakovic@grignon.inra.fr
}

consists of an N-terminal transmembrane loop (of variable size), followed by a cytosolic catalytic domain that contains the ATP-binding site and a C-terminal tail of autophosphorylatable tyrosine residues [5]. A distinctive feature of these enzymes is that they use a structural motif known as the Ploop (Walker motifs A and B) to constitute their active site. This feature is shared by only a few bacterial protein-kinases [8], whereas in Eukarya, Walker motifs are found in many ATP/GTPases [9], but not in protein kinases. In Firmicutes, the canonic BY-kinase gene has been split in two, encoding separately the transmembrane domain and the cytosolic kinase, both of which maintain a tight functional interaction despite being separated to independent polypeptide chains (Fig. 1). The cause and functional consequences of this separation remain unclear, although some new leads have appeared in recent studies and these will be discussed in the following chapter.

In the first phase of research on BY-kinases, since their discovery in 1996 and up to 2003, these enzymes were considered exclusively as autophosphorylating kinases. Initially, BY-kinase autophosphorylation has been linked to the functional role of these enzymes in exopolysaccharide production in several bacterial systems that were studied, since they are usually encoded by genes in the large operons involved in biosynthesis and export of sugar polymers [10]. One of the most exhaustively studied systems concerning BY-kinase implication in exopolysaccharide synthesis is the Proteobacteria model organism Escherichia coli. E. coli possesses two BY-kinases: Wzc encoded a gene in the operon which participates in the biosynthesis of polysaccharide polymers [11] 


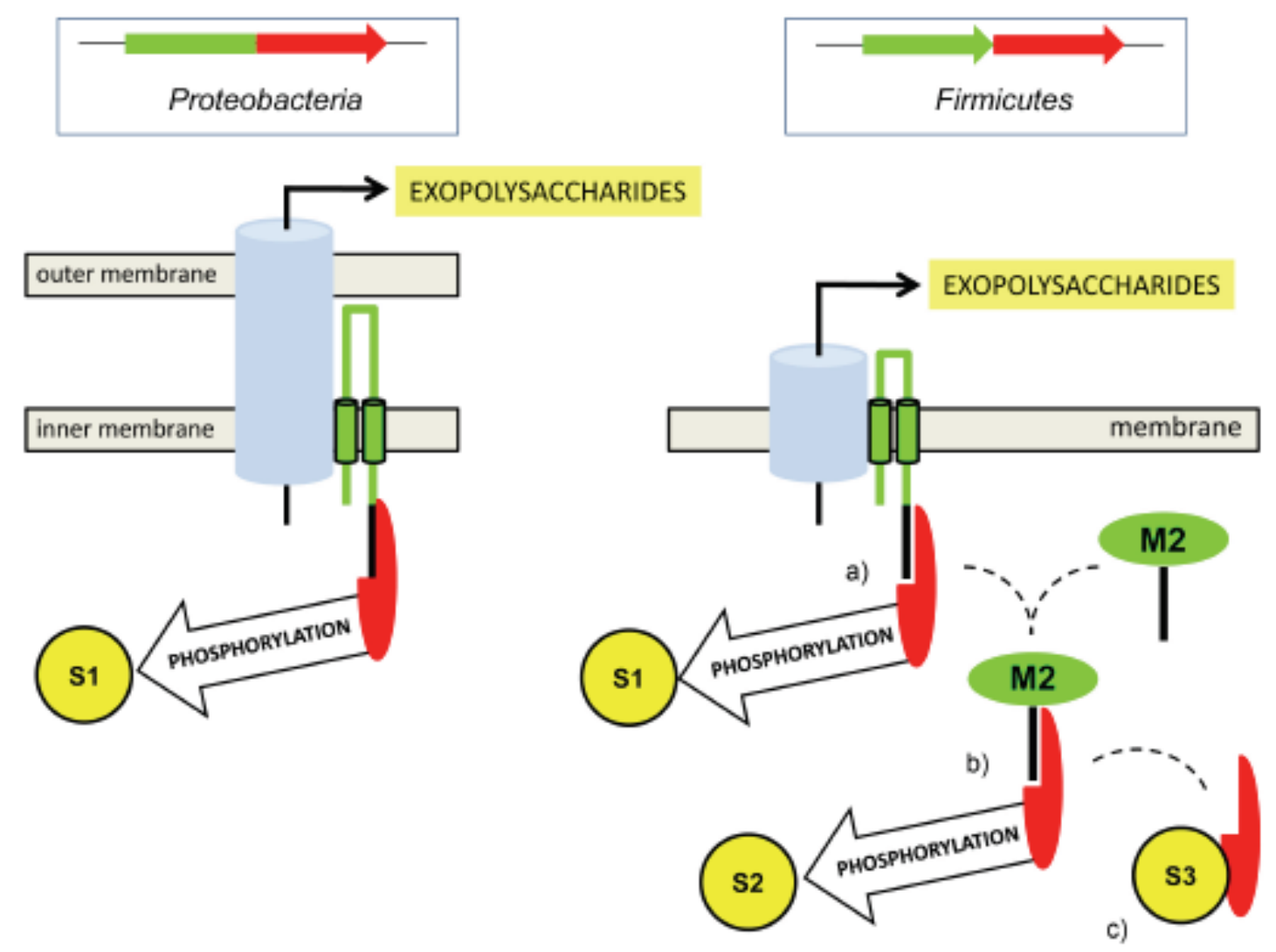

Fig. (1). Schematic representation of the two archetypal BY-kinase architectures. Proteobacteria have the two functional domains; transmembrane (green) and kinase (red) encoded by a single gene, and in Firmicutes these domains are independent proteins, encoded by separate genes. BY-kinases in both types of bacteria interact with the exopolysaccharide synthesis and translocation machinery (light blue), which is more complex in Proteobacteria where it has to traverse 2 membranes. The C-terminal end of the transmembrane domain that activates the kinase domain is represented in black. In Proteobacteria, this fragment is linked covalently to the kinase domain, and the active kinase domain is thus capable of phosphorylating the full set of its endogenous substrates (S1). In Firmicutes, the kinase is also activated by the Cterminal fragment of the transmembrane modulator, and can thus phosphorylate a set of physiological substrates (S1), as shown in case a). However, the kinase is free to dissociate from this modulator and meet other proteins that can accomplish this function (M2), and perhaps change its specificity towards another set of substrates (S2), as illustrated in case b). Finally, recent data indicate that the kinase can also colocalize with some of its substrates (S3), case c), although the physiological role of this co-localization is not clear at present.

and Etk, encoded by a gene in the G4C operon which is required for formation of group 4 capsule (G4C) polysaccharide [12]. It has been demonstrated that presence of Wzc and Etk is essential for synthesis of corresponding extracellular polysaccharides. More to the point, the auto-phosphorylation of tyrosine residues in the C-termini of these BY-kinases is the key feature in the assembly of capsular polysaccharides. Both the phosphorylated and the non-phosphorylated forms of Wzc are essential for polysaccharide polymer production $[13,14]$, and the current hypothesis is that the cycling between phosphorylated and non-phosphorylated form of Wzc allows the polysaccharide polymer synthesis to proceed correctly. Accordingly, Wzc and Etk have been classified as polysaccharide co-polymerases. In their co-polymerase capacity, these BY-kinases have been shown to influence the amount of polysaccharides as well as the length of the produced polymer probably via an interaction with a polysaccharide polymerase Wzy [14-16]. An interesting observation from the evolutionary standpoint is that there are members of the polysaccharide co-polymerase family which contain the transmembrane domain, but have no cytosolic kinase domain
[6]. A similar system has been described in Firmicutes, where the most extensively studied system with respect to exopolysaccharide production is probably that of Streptococcus pneumoniae. The autophosphorylating BY-kinase CpsD is essential for capsular polysaccharide biosynthesis in $S$. pneumoniae, where it regulates the amount of the synthesized capsular polysaccharide, which in turn affects the attachment of the bacteria to the host cells and contributes to the infection process $[17,18]$. Similar processes have been described in a number of other bacteria [5]. An interesting hypothesis has been put forward by Cuthbertson et al. [6] who argue that co-polymerases may simply influence the amount of produced polysaccharides by serving as molecular scaffolds for the other members of the translocon. The detailed mechanism of the functional interaction between polymerases and co-polymerases has not been entirely pinpointed, but considerable efforts are currently directed at this question.

After the initial link between BY-kinase autophosphorylation and exopolysaccharide production had been firmly 
established, this field took a new direction in the year 2003, when several teams simultaneously published reports that BY-kinases can phosphorylate other endogenous proteins substrates on tyrosine residues [19-21]. In each reported case the substrate activity was regulated by BY-kinase dependent phosphorylation (Fig. 2). E. coli Etk was found to phosphorylate $\mathrm{RpoH}$ (an alternative heat shock sigma factor) and an anti-sigma factor RseA and thus participate in triggering the heat shock response [19]. Its paralogue, Wzc [20], and a BY-kinase orthologue from Bacillus subtilis, PtkA [21], were found to phosphorylate UDP-glucose dehydrogenases in their respective organisms. The molecular mechanism of activation of UDP-glucose dehydrogenases by BY-kinase dependent tyrosine phosphorylation has been studied in molecular detail [22-24], and in the process, Etk from $E$ coli has also been found to phosphorylate the cognate UDP-glucose dehydrogenases [23]. Although the mechanisms of UDPglucose dehydrogenases activation are arguably not identical in E. coli [23] and B. subtilis [24], they both involve precise positioning of the phosphorylated tyrosine with respect to the bound substrate in the enzyme active site. Moreover, the investigation of the activation mechanism in E. coli has shed light on two parallel signal transduction pathways that intersect at the level of UDP-glucose dehydrogenase phosphorylation; one involving Wzc and the colanic acid synthesis and the other Etk and polymyxin resistance [23]. Examples of BY-kinases phosphorylating enzymes involved in the synthesis of sugar polymers have rapidly emerged in other bacterial systems. S. thermophilus phosphoglycosyltransferase EpsE is phosphorylated and activated by the cognate BYkinase EpsD [25], Staphylococcus aureus BY-kinase Cap5B2 can phosphorylate and activate an endogenous
UDP-acetyl-mannosamine dehydrogenase Cap5O [26], whereas tyrosine-phosphorylation of Klebsiella pneumoniae undecaprenolphosphate glycosyltransferase WcaJ has been reported as necessary for capsular polysaccharide synthesis [27]. The emergent phosphorylation networks based on BYkinases and their substrates hold promise of significant complexity. So far only one case of a single substrate phosphorylated by several kinases has been reported: E. coli Ugd phosphorylated by Wzc and Etk [23]. However, the examples of one kinase phosphorylating several substrates are plenty: $E$. coli Etk phosphorylates RpoH, RseA [19] and Ugd [23], and B. subtilis PtkA phosphorylates UDP-glucose dehydrogenases Ugd and TuaD [21], as well as single-stranded DNA-binding proteins SsbA and SsbB [28]. Phosphorylation of single-stranded DNA-binding proteins by BY-kinases, which results in increased affinity for DNA, hinted at an interesting possibility that BY-kinases migh in fact be extensively involved in regulating the DNA metabolism. B. subtilis cells devoid of PtkA exhibited a complex pleiotropic phenotype with defects in the cell cycle, initiation of the DNA replication and chromosome distribution [29]. In addition to $\mathrm{SsbA}$ and $\mathrm{SsbB}$, PtkA phosphorylates and regulates the activity of other proteins involved in B. subtilis singlestanded DNA metabolism, which may account for the complex phenotype of the $\triangle p t k A$ strain (Jers \& Mijakovic, unpublished results). In E. coli, the BY-kinase Wzc was found to phosphorylate integrase proteins (Int) of coliphages HK022 and $\lambda$. Overexpression of Wzc in tyrosinephosphatase deficient background resulted in a significantly reduced lysogenization, indicating that phosphorylation of Int down-regulates its activity [30].

capsule production

- attachment to host cells

- infection determinant

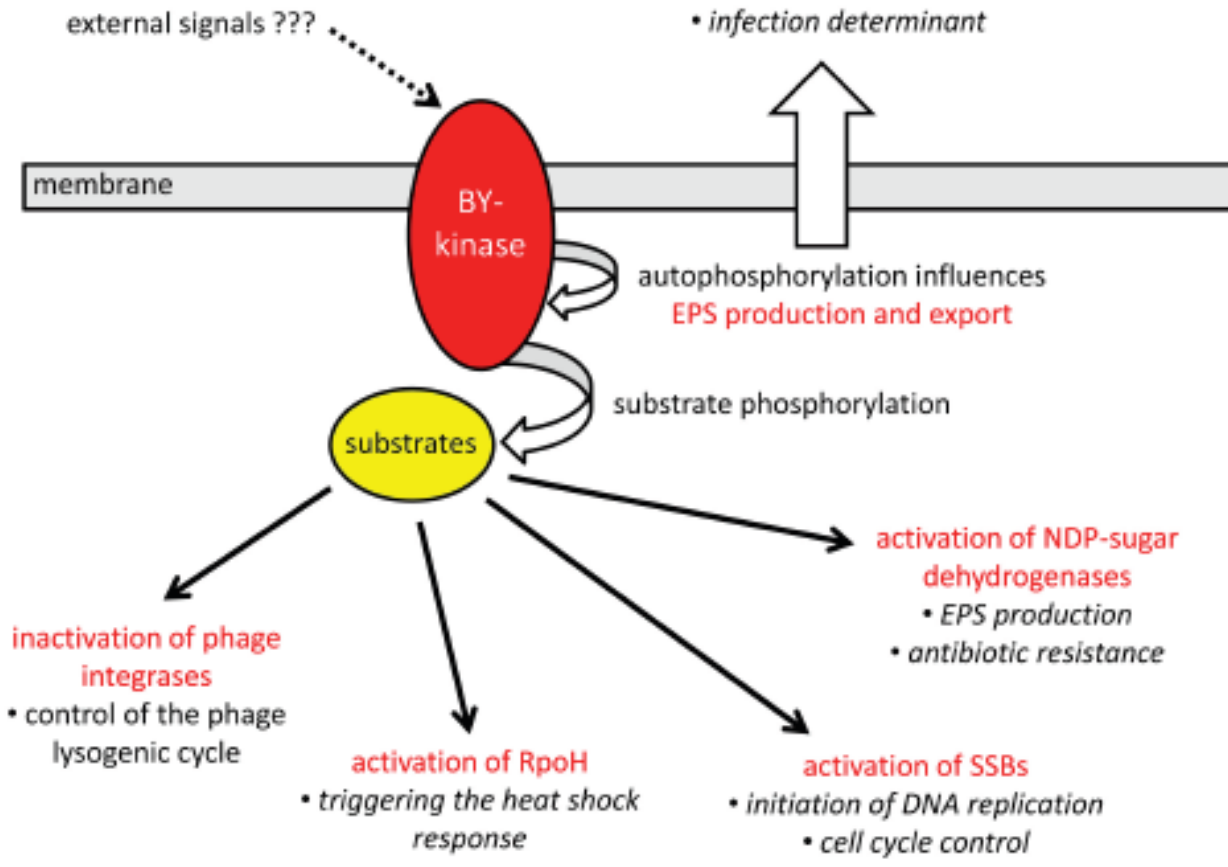

Fig. (2). Schematic representation of the physiological and regulatory roles of BY-kinases. BY-kinase autophosphorylation controls the involvement of these enzymes in the synthesis and translocation of sugar polymers. In addition, BY-kinases phosphorylate a number of intracellular substrates. Only well-studied cases are presented here, with clearly documented consequences for bacterial physiology. 
Following the finding that heat shock response in E. coli is modulated by the activity of the BY-kinase Etk [19], a link between tyrosine phosphorylation and heat shock was also published for B. subtilis. A protein with no homology to canonical BY-kinases, McsB, was found to phosphorylate CtsR, repressor of the heat-shock genes in the presence of a modulator protein McsA [31]. By consequence, the tyrosinephosphorylated CtsR was reported to release its target DNA. McsB was also found to act as a regulated adaptor protein for $\mathrm{ClpCP}$, the protease complex responsible for the degradation of CtsR [32]. The active, phosphorylated kinase McsB interacts with both CtsR and ClpC, and targets CtsR to the ClpCP complex. Finally, in 2009 McsB seems to have been taken off the list of tyrosine kinases, and its status revised to that of an arginine kinase [33]. It would be interesting to see whether this kinase has a dual specificity, or the discrepancies between the two reports were due to different detection methods. This by no means diminished the importance of previous findings that McsB functionally interacts with $\mathrm{CtsR}$ and ClpCP. At the same time, this new finding opened a new chapter of bacterial protein phosphorylation at arginine residues.

\section{PROTEOBACTERIA VS. FIRMICUTES: DIFFERENT STRUCTURES FOR DIFFERENT REGULATORY STRATEGIES?}

Important advances in the field of bacterial proteintyrosine kinases were made recently in terms of structural studies. The Walker motifs found in BY-kinases, and the overall sequence homology, had suggested that these proteins might structurally resemble bacterial ATPases such as MinD and Soj [5]. The first structural insights came from the low resolution structure (14 $\AA$ ) of $E$. coli BY-kinase Wzc in complex with the capsular polysaccharide translocon Wza [34]. It revealed a Wzc tetramer that oligomerises via the periplasmic domains, and with its cytosolic kinase domains freely protruding into the cytosol (not interacting with each other). The functional insights derived from this structure suggested that Wzc regulates export by triggering an open/active conformation of the translocase Wza. More recently, two high resolution structures of BY-kinases were published: Etk from E. coli [35] representing the Proteobacteria-type BY-kinase architecture, and CapA/B from $S$. aureus [36] representing the Firmicutes-type BY-kinase architecture. These structures provided insights into the functional role of several conserved features. In a previous biochemical study it was demonstrated that Etk homologue Wzc is activated through a two-step mechanism involving a conserved tyrosine in the active site and the C-terminal tyrosine cluster [37]. The structure data combined with biochemical and in silico analysis revealed a novel mechanism by which the first activation steps proceeds, showing that the conserved tyrosine side chain points into the active site thereby blocking activity. Upon autophosphorylation, the negatively charged phosphotyrosine rotates out of the active site and is stabilized by interaction with an arginine residue [35,38]. In Firmicutes, where the kinase domain is separated in a distinct polypeptide chain, it has been shown that the interaction with the remaining transmembrane domain is necessary for the kinase activity [21,39]. The structure of $S$. aureus CapB demonstrated that the activation proceeds, at least in part, by the fact that the C-terminal part of the transmembrane modulator (protruding into the cytosol) stabilizes ATP binding by hydrophobic sandwich interaction between the adenine ring and a phenylalanine residue of the modulator protein [36]. In case of CapB, the non-phosphorylated kinase monomers, as opposed to the case of Wzc, associated in an octameric ring structure, anchored to the membrane via interaction with the transmembrane modulator CapA. Notably, one of the tyrosine residues in the $\mathrm{C}$-terminal tyrosine cluster was found bound to the active site of the neighbouring subunit, suggesting an inter-molecular phosphorylation mechanism. The fact that no tyrosine residue in the cluster is preferentially phosphorylated reflects a high degree of flexibility in that region. An important conclusion from this study was that autophosphorylation of $\mathrm{CapB}$ is expected to induce dissociation of the ring structure, by a conformational change that disrupts the amino acid contacts at the interaction interface, while maintaining the interaction between individual CapB-CapA couples. The two BY-kinase structures also provided a rationale for the substrate specificity of Tyr vs Ser/Thr kinases and the difference between BY-kinases and the structurally highly similar ATPases such as MinD [40].

The resolved structures of BY-kinases have rekindled interest in a decade-old question, why are there distinct Proteobacteria- and Firmicutes-type architectures; one with a single-polypeptide chain, and the other with two functional domains (transmembrane and kinase) split in separate proteins (Fig. 1). BY-kinases in Proteobacteria have a large extracellular (periplasmic) loop that allows them to interact with the polysaccharide translocation machinery [6], and this loop is significantly shorter in Firmicutes which lack the outer membrane and all corresponding structures. However, both types of BY-kinases are perfectly capable of phosphorylating various intra-cellular protein substrates, so this particular activity (substrate phosphorylation and recognition of multiple substrates) does not seem to be linked with any one of the two particular architectures. The structural study on CapB indicated that upon octamer dissociation, each individual BY-kinase molecule remains in contact with its transmembrane modulator CapA, and were it ever to separate from it, it would become effectively inactive as a kinase, due to destabilization of the ATP-binding site [36]. And yet the fact remains, BY-kinases in Firmicutes have evolved with an option to dissociate from their transmembrane modulators, which makes it rather difficult to imagine that they never do so. Our group has recently performed localization studies on the BY-kinase PtkA in B. subtilis using fluorescent protein fusions, and we were able to demonstrate that PtkA colocalizes with its transmembrane modulator TkmA under certain growth conditions, but we were equally able to identify growth conditions where PtkA dissociates from TkmA and becomes prevalently cytosolic (Jers \& Mijakovic, unpublished results). In some cases PtkA also co-localized with some of its newly identified proteins substrates. We further demonstrated that PtkA can interact with other cytosolic proteins that are not its substrates but can modulate its kinase activity (Shi, Noirot-Gros \& Mijakovic, unpublished results). This incited us to speculate that BY-kinases in Firmicutes might have a separated kinase domain in order to make it accessible to other modulators, and therefore participate in signaling pathways other than the principal one that starts at 
the cell membrane, with the classical BY-kinase transmembrane domain. Arguments to support our hypothesis are admittedly not abundant at the moment, but we are confident that further studies in this direction will bring about another important turning point in the story of BY-kinases.

\section{DETECTING PHOSPHO-TYROSINES: DEVELOP- MENTS IN MASS SPECTROMETRY PHOSPHOPRO- TEOMICS}

In Eukarya, protein-tyrosine phosphorylation only accounts for approximately $0.05 \%$ of the total cellular protein phosphorylation, even though almost one fifth of the cellular kinome encodes protein-tyrosine kinases [41]. Despite the average scarcity in terms of occupancy of phosphorylation sites, protein-tyrosine phosphorylation plays key roles in eukaryal signal transduction, cell differentiation and growth. In Bacteria, occupancy of protein-tyrosine phosphorylation sites is also very low, to the extent that this type of phosphorylation has not been identified at all by traditional proteomics approaches using 2D-gels and low-resolution mass spectrometry [42]. Only recently, the advent of gel-free analysis coupled to high-resolution mass spectrometry enabled us to systematically detect phospho-tyrosines in bacterial phosphoproteomes [27, 43-45]. In terms of the number of identified phosphorylation sites, phospho-tyrosines account for $3-10 \%$ of published bacterial phosphoproteomes and this is a considerable over-representation compared to the eukaryal systems. Nevertheless, in terms of actual site occupancy tyrosine phosphorylation remains very low, and the signals of tyrosine-phosphorylated peptides during mass spectrometry analysis are as a rule difficult to detect due to ion suppression effects from a comparatively high background of non-phosphorylated peptides. Therefore, enrichment techniques for phosphotyrosine-containing peptides preceding mass spectrometry analysis represent a promising area for improvement. In all phosphoproteomic studies performed in bacteria so far, phosphopeptides were enriched by methods not distinguishing between various phosphoesters (phospho-serine, -threonine and -tyrosine), such as using strong cationic exchange, titanium-oxide or immobilized metal affinity chromatography. Anti-phosphoamino acid antibodies against phospho-serine/threonine and to lesser extent against phospho-tyrosine are infamous for somewhat relaxed specificity, due to the fact that antibodies recognize a stretch of 10-11 amino acids and a single phosphoamino acid is not an ideal epitope. While this may represent a challenge for specific detection by Western blots, in terms of phosphopeptide enrichment from crude protein extracts antibodies can be a useful tool. In studies performed on eukaryal systems, the 4G10 and the PY100 antibodies were found to be the most efficient for the immune-affinity enrichment of phosphotyrosine proteins and peptides, and a combined use of the two antibodies was recommended to obtain the best result [46]. It seems therefore that a viable route towards global tyrosine phosphorylation studies in bacteria might imply antibody based enrichment prior to mass spectrometry analysis. In terms of cellular signaling, protein-tyrosine phosphorylation is a dynamic event and quantitative and time resolved analytical approaches are prerequisite to understanding its physiological role; even more so bearing in mind the short generation time of bacteria. In quantitative phosphoproteomics, stable isotope labeling of cell cultures with amino acids (SILAC) provides reliable means of relative quantification of phosphotyrosines [47]. However, SILAC-based quantification imposes some limitations in terms of choice of growth medium. Alternative approaches rely on labeling after the growth phase, thus not influencing the growth conditions, such as for example the dimethylation of peptides with stable isotopes [48]. In parallel to mass spectrometry-based phosphoproteomics, unrelated approaches are being developed to study protein-tyrosine phosphorylation. They may lack the broadness of the global phosphoproteomic approaches, but may have other advantages in terms of time resolved detection of specific phosphorylation events. For example, electrochemical biosensors have been developed to monitor specific tyrosine kinase activities (and screening of inhibitors) in vitro [49]. In this particular study, the authors have used specific biotinylated substrate peptides immobilized on a streptavidin-coated indium-tin oxide electrode surface. The phosphorylation is detected using an ATP analogue adenosine gamma-thio triphosphate. After kinasecatalysed thio-phosphorylation of the immobilized peptides, the electrode surface was exposed to gold nanoparticles and the signals were thereafter detected by voltametry, using gold-chloride electrochemistry. This method seems particularly promising for in vitro screening of specific tyrosinekinase inhibitors, and other developments in nanotechnology biosensors may hold a promise for applications allowing in vivo studies of signal transduction in microorganisms.

\section{PERSPECTIVES}

Site-specific bacterial phosphoproteomes are being published at an increasing pace [27, 43-45], and with the high accuracy mass spectrometry becoming more accessible we may reasonably expect to identify large numbers of BYkinase substrates in various bacterial organisms. At present, bioinformatic predictors of protein phosphorylation sites are available. Neural-network algorithms, such as NetPhosBac [50], trained with available datasets on experimentally identified phosphorylation sites, can predict serine/threonine phosphorylation based on sequence homology and conservation of sites. Alternative predictors are the ones based on local structural disorder, such as DISPHOS [51]. At present, the number of identified sites of protein-tyrosine phosphorylation in bacterial proteins is not sufficient to envisage the construction of homology-based predictors such as NetPhosBac, but approaches based on local structural disorder seem more promising. In particular, weak conservation of bacterial phosphoproteomes on the phosphorylation site level is an argument in favour of developing clade-specific predictors, rather than global bacterial predictors. Upon examination of published tyrosine phosphorylation sites assigned to a specific kinase, as for example Ugd, TuaD, SsbA and SsbB proteins phosphorylated by PtkA in B. subtilis [21,28], no clear kinase recognition motif has emerged in the immediate surroundings of the phosphorylated residues. It is therefore plausible to assume that BY-kinases, much like their cognate phosphotyrosine-protein phosphatases [52], recognize structural motifs distant from the phosphorylated(able) residue on which they act. One big remaining question concerning the regulatory role of BY-kinases is the regulation of their kinase activity. BY-kinases described in recent structural 
studies offer a snapshot of a constitutively active kinase, but we cannot be certain that this is indeed the case in vivo. The presence of large extracellular loops, by analogy to eukaryal systems, suggests the existence of extracellular signals that might trigger kinase activity. However, in Proteobacteria these extracellular loops are heavily involved in interactions with polysaccharide translocation machinery, and it is questionable whether they may form a real signal acceptor site. In bacteria in general, our knowledge on extracellular signals that trigger the activity of cytosolic serine/threonine and tyrosine kinases is very limited [53], but this may simply reflect our lack of investigative approaches concerning this part of the signaling cascade. The amount of data gathered on BY-kinases is at present not extensive enough to allow indepth modeling of tyrosine-phosphorylation-based regulatory networks. However, the available elements on specific substrate activation should soon allow the construction of simple Boolean-type models, integrating all known BYkinase interactants. Since tyrosine phosphorylation is a dynamic event; it may be justified to invest some efforts in quantitative, time-resolved analysis of BY-kinase cellular functions, and create dynamic models describing the behavior of these regulatory loops. These efforts may be particularly well justified by the relatively high connectivity of BYkinases in Firmicutes, with a large number of substrates and alternative modulators.

Recent reviews concerning BY-kinases and bacterial protein phosphorylation in general [54,55] point out the promise they hold in terms of strategies for battling infective diseases caused by bacterial pathogens. The main advantage of BY-kinases in this respect is the fact that they have no eukaryal homologues, so their specific inhibitors are less likely to affect the host than for example the inhibitors of Hanks type serine/threonine kinases, which are present both in Bacteria and Eukarya. However, as discussed above, BYkinases seem to be quite promiscuous in terms of choosing their substrates. Along these lines, Lin et al. [27] justifiably argue that site-specific knowledge concerning the substrates regulated by BY-kinases might provide more specific targets for inhibitory drugs. There is nevertheless an agreement in the field that inhibiting bacterial tyrosine phosphorylation is a promising venue in developing new therapeutics, and considerable efforts are already committed to this approach.

\section{ACKNOWLEDGEMENTS}

This work was supported by grants from the Lundbeckfonden and the Institut National de Recherche Agronomique (INRA) to IM and a $\mathrm{PhD}$ stipend from the Technical University of Denmark (DTU) to CJ.

\section{References}

[1] Abu-Qarn, M.; Eichler, J. and Sharon N. Not just for Eukarya anymore: protein glycosylation in Bacteria and Archaea. Curr. Opin. Struct. Biol., 2008, 18, 544-550.

[2] Lauber, M.A.; Running, W.E. and Reilly, J.P. B. subtilis ribosomal proteins: structural homology and post-translational modifications. J. Proteome Res., 2009, 8, 4193-4206.

[3] Duclos, B.; Grangeasse, C.; Vaganay, E.; Riberty, M. and Cozzone, A.J. Autophosphorylation of a bacterial protein at tyrosine. J. Mol. Biol., 1996, 259, 891-895.

[4] Grangeasse, C.; Doublet, P.; Vaganay, E.; Vincent, C.; Deléage, G.; Duclos, B. and Cozzone, A.J. Characterization of a bacterial gene encoding an autophosphorylating protein tyrosine kinase. Gene, 1997, 204, 259-265.

[5] Grangeasse, C.; Cozzone, A.J.; Deutscher, J. and Mijakovic, I. Tyrosine phosphorylation: an emerging regulatory device of bacterial physiology. Trends Biochem. Sci., 2007, 32, 86-94.

[6] Cuthbertson, L.; Mainprize, I.L.; Naismith, J.H. and Whitfield, C. Pivotal roles of the outer membrane polysaccharide export and polysaccharide copolymerase protein families in export of extracellular polysaccharides in Gram-negative bacteria. Microbiol. Mol. Biol. Rev., 2009, 73, 155-177.

[7] Jadeau, F.; Bechet, E.; Cozzone, A.J.; Deléage, G.; Grangeasse, C. and Combet, C. Identification of the idiosyncratic bacterial protein tyrosine kinase (BY-kinase) family signature. Bioinformatics, 2008, 24, 2427-2430.

[8] Mijakovic, I.; Poncet, S.; Galinier, A.; Monedero, V.; Fieulaine; S., Janin, J.; Nessler, S.; Marquez, H.-A.; Scheffzek, K.; Hasenbein, S.; Hengstenberg, W. and Deutscher, J. Pyrophosphate-producing protein dephosphorylation by HPr kinase/phosphorylase: a relic of early life? Proc. Natl. Acad. Sci. USA, 2002, 99, 13442-13347.

[9] Patel, S. and Latterich, M. The AAA team: related ATPases with diverse functions. Trends Cell. Biol., 1998, 8, 65-71.

[10] Whitfied, C. Biosynthesis and assembly of capsular polysaccharides in Escherichia coli. Annu. Rev. Biochem., 2006, 75, 39-68.

[11] Vincent, C.; Doublet, P.; Grangeasse, C.; Vaganay, E.; Cozzone, A.J. and Duclos, B. Cells of Escherichia coli contain a proteintyrosine kinase, Wzc, and a phosphotyrosine-protein phosphatase, Wzb. J. Bacteriol., 1999, 181, 3472-3477.

[12] Peleg, A.; Shifrin, Y.; Ilan, O.; Nadler-Yona, C.; Nov, S.; Koby, S.; Baruch, K.; Altuvia, S.; Elgrably-Weiss, M.; Abe, C.M.; Knutton, S.; Saper, M.A. and Rosenshine, I. Identification of an Escherichia coli operon required for formation of the O-antigen capsule. J. Bacteriol., 2005, 187, 5259-5266.

[13] Paiment, A.; Hocking, J. and Whitfield, C. Impact of phosphorylation of specific residues in the tyrosine autokinase, Wzc, on its activity in assembly of group 1 capsules in Escherichia coli. J. Bacteriol., 2002, 184, 6437-6447.

[14] Obadia, B.; Lacour, S.; Doublet, P.; Baubichon-Cortay, H.; Cozzone, A.J. and Grangeasse, C. Influence of tyrosine-kinase Wzc activity on colanic acid production in Escherichia coli $\mathrm{K} 12$ cells. $J$. Mol. Biol., 2007, 367, 42-53.

[15] Tocilj, A.; Munger, C.; Proteau, A.; Morona, R.; Purins, L. Ajamian, E.; Wagner, J.; Papadopoulos, M.; Van Den Bosch, L.; Rubinstein, J.L.; Féthière, J.; Matte, A. and Cygler, M. Bacterial polysaccharide co-polymerases share a common framework for control of polymer length. Nat. Struct. Mol. Biol., 2008, 15, 130138.

[16] Whitfield, C. and Larue, K. Stop and go: regulation of chain length in the biosynthesis of bacterial polysaccharides. Nat. Struct. Mol. Biol., 2008, 15, 121-123.

[17] Morona, J.K.; Morona, R. and Paton, J.C. Attachment of capsular polysaccharide to the cell wall of Streptococcus pneumoniae type 2 is required for invasive disease. Proc. Natl. Acad. Sci. USA, 2006, $103,8505-8510$.

[18] Morona, J.K.; Miller, D.C.; Morona, R. and Paton, J.C. The effect that mutations in the conserved capsular polysaccharide biosynthesis genes cpsA, cpsB, and cpsD have on virulence of Streptococcus pneumoniae. J. Infect. Dis., 2004, 189, 1905-1913.

[19] Klein, G.; Dartigalongue, C. and Raina, S. Phosphorylationmediated regulation of heat shock response in Escherichia coli. Mol. Microbiol., 2003, 48, 269-285.

[20] Grangeasse, C.; Obadia, B.; Mijakovic, I.; Deutscher, J.; Cozzone, A.J. and Doublet, P. Autophosphorylation of the Escherichia coli protein kinase Wzc regulates tyrosine phosphorylation of Ugd, a UDP-glucose dehydrogenase. J. Biol. Chem., 2003, 278, 3932339329.

[21] Mijakovic, I.; Poncet, S.; Boël, G.; Mazé, A.; Gillet, S.; Jamet, E.; Decottignies, P.; Grangeasse, C.; Doublet, P.; Le Maréchal, P. and Deutscher, J. Transmembrane modulator-dependent bacterial tyrosine kinase activates UDP-glucose dehydrogenases. EMBO J., 2003, 22, 4709-4718.

[22] Mijakovic, I.; Petranovic, D. and Deutscher, J. How tyrosine phosphorylation affects the UDP-glucose dehydrogenase activity of $B a$ cillus subtilis YwqF. J. Mol. Microbiol. Biotechnol., 2004, 8, 1925 .

[23] Lacour, S.; Bechet, E.; Cozzone, A.J.; Mijakovic, I. and Grangeasse, C. Tyrosine phosphorylation of the UDP-glucose de- 
hydrogenase of Escherichia coli is at the crossroads of colanic acid synthesis and polymyxin resistance. PLoS One, 2008, 3, e3053.

[24] Petranovic, D.; Grangeasse, C.; Macek, B.; Abdillatef, M.; Gueguen-Chaignon, V.; Nessler, S.; Deutscher, J. and Mijakovic, I. Activation of Bacillus subtilis Ugd by the BY-kinase PtkA proceeds via phosphorylation of its residue tyrosine 70. J. Mol. Microbiol. Biotechnol., 2009, 17, 83-89.

[25] Minic, Z.; Marie, C.; Delorme, C.; Faurie, J.M.; Mercier, G.; Ehrlich, D. and Renault, P. Control of EpsE, the phosphoglycosyltransferase initiating exopolysaccharide synthesis in Streptococcus thermophilus, by EpsD tyrosine kinase. J. Bacteriol., 2007, 189, 1351-1357.

[26] Soulat, D.; Grangeasse, C.; Vaganay, E.; Cozzone, A.J. and Duclos, B. UDP-acetyl-mannosamine dehydrogenase is an endogenous protein substrate of Staphylococcus aureus protein-tyrosine kinase activity. J. Mol. Microbiol. Biotechnol., 2007, 13, 45-54.

[27] Lin, M.H.; Hsu, T.L.; Lin, S.Y.; Pan, Y.J.; Jan, J.T.; Wang, J.T.; Khoo, K.H. and Wu, S.H. Phosphoproteomics of Klebsiella pneumonia NTUH-K2044 reveals a tight link between tyrosine phosphorylation and virulence. Mol. Cell. Proteom., 2009, in press, PMID: 19696081.

[28] Mijakovic, I.; Petranovic, D.; Macek, B.; Cepo, T.; Mann, M.; Davies, J.; Jensen, P.R.; and Vujaklija, D. Bacterial single-stranded DNA-binding proteins are phosphorylated on tyrosine. Nucleic Acids Res., 2006, 34, 1588-1596.

[29] Petranovic, D.; Michelsen, O.; Zahradka, K.; Silva, C.; Petranovic, M.; Jensen, P.R. and Mijakovic, I. Bacillus subtilis strain deficient for the protein-tyrosine kinase PtkA exhibits impaired DNA replication. Mol. Microbiol., 2007, 63, 1797-1805.

[30] Kolot, M.; Gorovits, R.; Silberstein, N.; Fichtman, B. and Yagil, E. Phosphorylation of the integrase protein of coliphage HK022. Virology, 2008, 375, 383-390.

[31] Kirstein, J.; Zühlke, D.; Gerth, U.; Turgay, K. and Hecker, M. A tyrosine kinase and its activator control the activity of the CtsR heat shock repressor in B. subtilis. EMBO J., 2005, 24, 3435-3445.

[32] Kirstein, J.; Dougan, D.A.; Gerth, U.; Hecker, M. and Turgay, K. The tyrosine kinase McsB is a regulated adaptor protein for ClpCP. EMBO J., 2007, 26, 2061-2070.

[33] Fuhrmann, J.; Schmidt, A.; Spiess, S.; Lehner, A.; Turgay, K.; Mechtler, K.; Charpentier, E. and Clausen, T. McsB is a protein arginine kinase that phosphorylates and inhibits the heat-shock regulator CtsR. Science, 2009, 324, 1323-1327.

[34] Collins, R.F.; Beis, K.; Dong, C.; Botting, C.H.; McDonnell, C.; Ford, R.C.; Clarke, B.R.; Whitfield, C. and Naismith, J.H. The 3D structure of a periplasm-spanning platform required for assembly of group 1 capsular polysaccharides in Escherichia coli. Proc. Natl. Acad. Sci. USA, 2007, 104, 2390-2395.

[35] Lee, D.C.; Zheng, J.; She, Y.M. and Jia, Z. Structure of Escherichia coli tyrosine kinase Etk reveals a novel activation mechanism. EMBO J., 2008, 27, 1758-1766.

[36] Olivares-Illana, V.; Meyer, P.; Bechet, E.; Gueguen-Chaignon, V.; Soulat, D.; Lazereg-Riquier, S.; Mijakovic, I.; Deutscher, J.; Cozzone, A.J.; Laprévote, O.; Morera, S.; Grangeasse, C. and Nessler, $\mathrm{S}$. Structural basis for the regulation mechanism of the tyrosine kinase CapB from Staphylococcus aureus. PLoS Biol., 2008, 6, e143.

[37] Grangeasse, C.; Doublet, P. and Cozzone, A.J. Tyrosine phosphorylation of protein kinase Wzc from Escherichia coli K12 occurs through a two-step process. J. Biol. Chem., 2002, 277, 71277135.

[38] Lu, T.; Tan, H.; Lee, D.; Chen, G. and Jia, Z. New insights into the activation of Escherichia coli tyrosine kinase revealed by molecu- lar dynamics simulation and biochemical analysis. Biochemistry, 2009, 48, 7986-7995.

[39] Soulat, D.; Jault, J.M.; Duclos, B.; Geourjon, C.: Cozzone, A.J.; and Grangeasse, C. Staphylococcus aureus operates proteintyrosine phosphorylation through a specific mechanism. J. Biol. Chem., 2006, 281, 14048-14056.

[40] Lee, D.C. and Jia, Z. Emerging structural insights into bacterial tyrosine kinases. Trends Biochem. Sci., 2009, 34, 351-357.

[41] Blume-Jensen. P. and Hunter, T. Oncogenic kinase signaling. $\mathrm{Na}$ ture, 2001, 411, 355-365.

[42] Bendt, A.K.; Burkovski, A.; Schaffer, S.; Bott, M.; Farwick, M. and Hermann, T. Towards a phosphoproteome map of Corynebacterium glutamicum. Proteomics, 2003, 3, 1637-1646.

[43] Macek, B.; Mijakovic, I.; Olsen, J.V.; Gnad, F.; Kumar, C.; Jensen, P.R. and Mann, M. The serine/threonine/tyrosine phosphoproteome of the model bacterium Bacillus subtilis. Mol. Cell. Proteom., 2007, 6, 697-707.

[44] Macek, B.; Gnad, F.; Soufi, B.; Kumar, C.; Olsen, J.V.; Mijakovic, I. and Mann, M. Phosphoproteome analysis of E. coli reveals evolutionary conservation of bacterial Ser/Thr/Tyr phosphorylation. Mol. Cell. Proteom., 2008, 7, 299-307.

[45] Soufi, B.; Gnad, F.; Jensen, P.R.; Petranovic, D.; Mann, M.; Mijakovic, I. and Macek, B. The Ser/Thr/Tyr phosphoproteome of Lactococcus lactis IL1403 reveals multiply phosphorylated proteins. Proteomics, 2008, 8, 3486-3493.

[46] Lind, S.B.; Molin, M.; Savitski, M.M.; Emilsson, L.; Åström, J.; Hedberg, L., Adams, C.; Nielsen, M.L., Engström, A.; Elfineh, L.; Andersson, E.; Zubarev, R.A. and Pettersson, U. Immunoaffinity enrichments followed by mass spectrometric detection for studying global protein tyrosine phosphorylation. J. Prot. Res., 2008, 7, 2897-2910.

[47] Bantscheff, M.; Schirle, M.; Sweetman, G.; Rick, J. and Kuster, B. Quantitative mass spectrometry in proteomics: a critical review. Anal. Bioanal. Chem., 2007, 389, 1017-1031.

[48] Boersema, P.J.; Aye, T.T.; van Veen, T.A.; Heck, A.J. and Mohammed, S. Triplex protein quantification based on stable isotope labeling by peptide dimethylation applied to cell and tissue lysates. Proteomics, 2008, 8, 4624-4632.

[49] Kerman, K. and Kraatz, H.B. Electrochemical detection of protein tyrosine kinase-catalysed phosphorylation using gold nanoparticles. Biosens. Bioelectron., 2009, 24, 1484-1489.

[50] Miller, M.L.; Soufi, B.; Jers, C.; Blom, N.; Macek, B. and Mijakovic, I. NetPhosBac - a predictor for Ser/Thr phosphorylation sites in bacterial proteins. Proteomics, 2009, 9, 116-125.

[51] Iakoucheva, L.M.; Radivojac, P.; Brown, C.J.; O'Connor, T.R. Sikes, J.G.; Obradovic, Z. and , Dunker, A.K. Intrinsic disorder and protein phosphorylation. Nucleic Acids Res., 2004, 32, 1037-1049.

[52] Mijakovic, I.; Musumeci, L.; Tautz, L.; Petranovic, D.; Edwards, R.A.; Jensen, P.R.; Mustelin, T.; Deutscher, J. and Bottini N. In vitro characterization of the Bacillus subtilis protein tyrosine phosphatase YwqE. J. Bacteriol., 2005, 187, 3384-3390.

[53] Shah, I.M.; Laaberki, M.H.; Popham, D.L. and Dworkin, J. A eukaryotic-like Ser/Thr kinase signals bacteria to exit dormancy in response to peptidoglycan fragments. Cell, 2008, 135, 486-496.

[54] Grangeasse, C.; Terreux, R. and Nessler, S. Bacterial tyrosinekinases: structure-function analysis and therapeutic potential. Biochim. Biophys. Acta, 2009, in press, doi 10.1016/j.bbapap.2009.08.018

[55] Jers, C.; Soufi, B.; Grangeasse, C.; Deutscher, J. and Mijakovic, I. Phosphoproteomics in bacteria: towards a systemic understanding of bacterial phosphorylation networks. Exp. Rev. Proteom., 2008, $5,619-627$. 